\title{
Dissecting the immune cell landscape in hepatocellular carcinoma-are we understanding complexity?
}

\author{
Matthias Ocker ${ }^{1,2}$ \\ ${ }^{1}$ Charité University Medicine Berlin, Berlin, Germany; ${ }^{2}$ Translational Medicine \& Clinical Pharmacology, Boehringer Ingelheim Pharma GmbH \& \\ Co. KG, Ingelheim, Germany \\ Correspondence to: Prof. Dr. Matthias Ocker. Charité University Medicine Berlin, Charitéplatz 1, 10117 Berlin, Germany. \\ Email: matthias.ocker@charite.de. \\ Provenance and Peer Review: This article was commissioned and reviewed by the Section Editor Dr. Rui Liao (Department of Hepatobiliary Surgery, \\ The First Affiliated Hospital of Chongqing Medical University, Chongqing, China). \\ Comment on: Tang X, Shu Z, Zhang W, et al. Clinical significance of the immune cell landscape in hepatocellular carcinoma patients with different \\ degrees of fibrosis. Ann Transl Med 2019;7:528.
}

Submitted Feb 28, 2020. Accepted for publication Mar 26, 2020.

doi: $10.21037 /$ atm.2020.03.170

View this article at: http://dx.doi.org/10.21037/atm.2020.03.170

Despite its high global prevalence and increasing incidence, treatment of advanced hepatocellular carcinoma (HCC) remains unsatisfying. Since the introduction of the first multi-kinase inhibitor sorafenib more than 10 years ago, only incremental benefit has been achieved in first and second line settings resulting in a dismal five-year survival rate of only $1 \%$ (1). In addition to a high intrinsic resistance of HCC cells to cytotoxic agents, no clear oncogenic driver has been identified so far which could be used for targeted therapies (2). A reason for this could be the fact that HCC usually develops as a "disease within a disease", i.e., based on underlying chronic viral infections, chronic inflammatory conditions like NASH and the association with different degrees of fibrosis and cirrhosis. This pathophysiologic heterogeneity is commonly neglected when trying to identify novel predictive biomarkers for HCC (3), leading to only small and less comparable subpopulations.

Cancer immunotherapy (CIT) has evolved as a novel means to overcome these limitations. The development of immune checkpoint inhibitors like the anti-CTLA4 antibody ipilimumab or anti-PD1/PD-L1 antibodies like pembrolizumab, nivolumab or atezolizumab has dramatically changed the treatment landscape in various solid tumors and achieved long-lasting clinical responses in, e.g., melanoma or non-small cell lung cancer (NCSCLC) (4). Several CIT approaches have been studied in HCC but the achieved response rates of about $15-20 \%$ are considered only modest $(5,6)$. Considerable efforts are therefore taken to identify better predictive biomarkers beyond expression of PD-1/PD-L1 or tumor mutational burden. The commonly found chronic inflammatory conditions underlying HCC strongly impact on the immune cell activity needed for successful CIT and esp. cirrhosis has been associated with severe immune dysfunction. In a recent publication, Tang et al. therefore investigated the composition of the immune cell landscape in HCC in relation to its underlying fibrosis or cirrhosis to better understand which patients would benefit from established or novel CIT approaches (7).

The authors used two independent gene expression profiles (GEO, TCGA) and performed a bioinformatics analysis (CIBERSORT) to quantify relative levels immune cell types within the gene expression data sets. Results were then further correlated with survival analysis and clinical parameters. Significant differences were found between different stages of cirrhosis, dysplasia and HCC for $\mathrm{T}$ cell subpopulations, plasma cells and innate immune cells. In brief, the results show that multiple components are dysregulated during HCC formation and that both $\mathrm{T}$ cell immunity as well as myeloid cells are affected to create an immunosuppressive environment. In a second step, differentially expressed genes related to immune cell function were identified and correlated to overall survival (OS) and recurrence free survival (RFS) in the HCC 
subpopulation. Here, PVRIG (PVR related immunoglobulin domain-containing, CD122R) and FCER1A (Fc fragment of $\mathrm{IgE}$ receptor Ia) were found to be significantly associated with OS and RFS. Both genes are known to be associated to immune checkpoint inhibitors like CTLA4 or LAG3 or to NK or mast cell function (7-11). The study confirms previous results from Rohr-Udilova et al. but nicely expands the data by including fibrosis scores into the analyses (12).

These findings are of importance as they demonstrate that algorithms based on gene expression profiles (CIBERSORT) can identify immune phenotype subsets that may lead to the identification of potential novel predictive or prognostic biomarkers. While current CIT focuses largely on (reactivation of) $\mathrm{T}$ cell populations, the findings from Tang et al. indicate that also non-T cells might play an important tole in creating an immune evasive environment. In a recent publication, the role of $\mathrm{PD}-1$ in contributing to anti-tumor immunity was demonstrated also in myeloid cells in mice (13). Interestingly, this phenotype was associated with metabolic alterations in glycolysis and with hypercholesterolemia, indicating also a potential new link to NASH and HCC formation by myeloid cells depleted of PD-1. Yet, such data also imply that myeloid cells should be taken into consideration as a potential predictive biomarker also for current CIT. While direct evidence in HCC is still missing, both intratumoral and extratumoral macrophages and myeloid derived suppressor cells were correlated to poor response to immune checkpoint blockades in other tumors (5). Including such cell types in predictive biomarker panels may thus improve the so far mediocre response rates of CIT in HCC, too.

Some aspects also need critical attention. So far, only the retrospective database derived data is available which provides a good overall basis and allows hypothesis generation but may not be entirely representative for real life patient samples and data. Due to the early disease stages recruited into TCGA studies (patients often underwent curative surgery), only a small number of the approx. two hundred HCC cases available has metastatic or advanced disease stages. Interestingly, NAFLD was diagnosed only in less than $10 \%$ of cases while viral hepatitis (alone or in conjunction with other risk factors) was seen in about one third of cases. This underlines the importance to validate the obtained data in an independent and prospective study that needs to be adequately powered for small subgroup analyses and to account for the various underlying etiologies and the differential status of inflammation.
In summary, such studies create important unbiased data that identify potential new biomarkers that are of importance to improving the outcome of patients with HCC, but may need additional clinical validation and real life data from clinical trials.

\section{Acknowledgments}

Funding: None.

\section{Footnote}

Conflicts of Interest: The author has completed the ICMJE uniform disclosure form (available at http://dx.doi. org/10.21037/atm.2020.03.170). The author has no conflicts of interest to declare.

Ethical Statement: The author is accountable for all aspects of the work in ensuring that questions related to the accuracy or integrity of any part of the work are appropriately investigated and resolved.

Open Access Statement: This is an Open Access article distributed in accordance with the Creative Commons Attribution-NonCommercial-NoDerivs 4.0 International License (CC BY-NC-ND 4.0), which permits the noncommercial replication and distribution of the article with the strict proviso that no changes or edits are made and the original work is properly cited (including links to both the formal publication through the relevant DOI and the license). See: https://creativecommons.org/licenses/by-nc-nd/4.0/.

\section{References}

1. Siegel RL, Miller KD, Jemal A. Cancer statistics, 2019. CA Cancer J Clin 2019;69:7-34.

2. Neureiter D, Stintzing S, Kiesslich T, et al. Hepatocellular carcinoma: Therapeutic advances in signaling, epigenetic and immune targets. World J Gastroenterol 2019;25:3136-50.

3. Ocker M. Biomarkers for hepatocellular carcinoma: What's new on the horizon? World J Gastroenterol 2018;24:3974-9.

4. Chae YK, Arya A, Iams W, et al. Current landscape and future of dual anti-CTLA4 and PD-1/PD-L1 blockade immunotherapy in cancer; lessons learned from clinical trials with melanoma and non-small cell lung cancer (NSCLC). J Immunother Cancer 2018;6:39. 
5. Tai D, Choo SP, Chew V. Rationale of Immunotherapy in Hepatocellular Carcinoma and Its Potential Biomarkers. Cancers (Basel) 2019. doi: 10.3390/cancers11121926.

6. Cheng H, Sun G, Chen H, et al. Trends in the treatment of advanced hepatocellular carcinoma: immune checkpoint blockade immunotherapy and related combination therapies. Am J Cancer Res 2019;9:1536-45.

7. Tang $X$, Shu Z, Zhang $W$, et al. Clinical significance of the immune cell landscape in hepatocellular carcinoma patients with different degrees of fibrosis. Ann Transl Med 2019;7:528.

8. Murter B, Pan X, Ophir E, et al. Mouse PVRIG Has CD8(+) T Cell-Specific Coinhibitory Functions and Dampens Antitumor Immunity. Cancer Immunol Res 2019;7:244-56.

9. Whelan S, Ophir E, Kotturi MF, et al. PVRIG and PVRL2 Are Induced in Cancer and Inhibit CD8(+) T-cell

Cite this article as: Ocker M. Dissecting the immune cell landscape in hepatocellular carcinoma-are we understanding complexity? Ann Transl Med 2020;8(11):666. doi: 10.21037/ atm.2020.03.170
Function. Cancer Immunol Res 2019;7:257-68.

10. Sanchez-Correa B, Valhondo I, Hassouneh F, et al. DNAM-1 and the TIGIT/PVRIG/TACTILE Axis: Novel Immune Checkpoints for Natural Killer CellBased Cancer Immunotherapy. Cancers (Basel) 2019. doi: 10.3390/cancers11060877.

11. Olivera A, Beaven MA, Metcalfe DD. Mast cells signal their importance in health and disease. J Allergy Clin Immunol 2018;142:381-93.

12. Rohr-Udilova N, Klinglmuller F, Schulte-Hermann R, et al. Deviations of the immune cell landscape between healthy liver and hepatocellular carcinoma. Sci Rep 2018;8:6220.

13. Strauss L, Mahmoud MAA, Weaver JD, et al. Targeted deletion of PD-1 in myeloid cells induces antitumor immunity. Sci Immunol 2020. doi: 10.1126/sciimmunol. aay1863. 